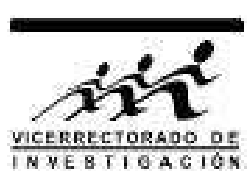

\title{
Estimación del nivel medio de bajamares de sicigias ordinarias en la bahía de Paracas
}

\author{
C. Jiménez ${ }^{* 1,2}$, M. Saavedra ${ }^{1,3}, J_{\text {N. Niquen }}^{1,4}$, J. Pasapera $^{1,4}$ y M. Calvo ${ }^{1}$ \\ ${ }^{1}$ Facultad de Ciencias Físicas, Universidad Nacional Mayor de San Marcos, Lima, Perú \\ ${ }^{2}$ Dirección de Hidrografía y Navegación, Callao, Perú \\ ${ }^{3}$ Servicio Nacional de Meteorología e Hidrología del Perú, Lima, Perú \\ ${ }^{4} \mathrm{HyO}$ Ingenieros S.A., Lima, Perú
}

Recibido 20 diciembre 2011 - Aceptado 30 marzo 2012

\begin{abstract}
La Tierra es un sistema dinámico y como tal es susceptible a la acción de eventos sísmicos y geológicos. Para muchos problemas de ciencias e ingeniería surge la necesidad de establecer un sistema de coordenadas tridimensional para realizar mediciones geodésicas. Con el avance de la geodesia satelital, en la actualidad es posible determinar las coordenadas horizontales de un punto sobre la superficie terrestre con errores menores a $1 \mathrm{~cm}$. El problema surge al tratar de obtener la coordenada vertical, ya que los satélites proporcionan alturas con referencia a un elipsoide, el cual no es una superficie equipotencial. Para obtener la coordenada vertical, se toma como referencia la superficie del geoide o nivel medio del mar. En el presente trabajo, se proporciona una metodología para estimar el nivel medio del mar en la bahía de Paracas, utilizando un sensor de nivel ultrasónico y un sistema de interface a la computadora. Los datos obtenidos son procesados digitalmente mediante herramientas de filtrado y transformada de Fourier para separar las componentes de frecuencia correspondientes a la marea y olas. Como resultado se obtiene un nivel de referencia con un error de $\pm 2 \mathrm{~cm}$, lo que es suficiente para muchas obras de ingeniería.
\end{abstract}

Palabras claves: nivel medio del mar, marea, oleaje.

\section{Estimation of average level of ordinary syzygy low tides in Paracas Bay}

The Earth is a dynamic system and as such is susceptible to the action of seismic and geological events. For many problems in science and engineering there is a need to establish a three-dimensional coordinate system for geodesic measurements. With the advancement of satellite geodesy, is currently possible to determine the horizontal coordinates of a point on the Earth's surface with errors less than one centimeter. The problem arises when trying to obtain the vertical coordinate, since satellites provide heights with reference to an ellipsoid, which is not an equipotential surface. For obtaining the vertical coordinate, the reference surface of the geoid or mean level sea is taken. In the present study, a methodology to determine the mean sea level in the Paracas Bay is provided, using an ultrasonic level sensor interfaced to a computer. The data are digitally processed using filtering tools and Fourier transform to separate the frequency components corresponding to the tide and surge. The result is a reference level with an error of $\pm 2 \mathrm{~cm}$, which is sufficient for many engineering works.

Keywords: Mean sea level, tide, surge.

La bahía de Paracas está ubicada en la provincia de Pisco, región Ica, en la parte central del Perú. Es un atractivo balneario costero, con modernas residencias, numerosos hoteles y su cálido clima, que la han convertido en uno de los principales destinos turísticos del país.
Durante el terremoto del 15 de agosto de 2007 con epicentro cercano a la ciudad de Pisco, muchas de las construcciones colapsaron. Según los datos geodésicos tomados por el Instituto Geofísico del Perú, IGP, después del sismo para un punto ubicado en Punta Paracas, la placa continental se movió hacia el mar 1.60

*cesarsud86@hotmail.com 
$\mathrm{m}$ en el plano horizontal y en la vertical se elevó 0.50 $\mathrm{m}$ [1. Además de esto se produjo la licuefacción de los suelos en algunos puntos de la ciudad. Lo que significa que toda medida u observación topográfica realizada con respecto a cualquier hito geodésico del IGP y de la Dirección de Hidrografía y Navegación, DHN, después del sismo no estará bien referenciada. En particular, el realizar la nivelación vertical con respecto al nivel medio de bajamares de sicigias ordinarias con respecto a estos hitos no se ajustará a la realidad física después del terremoto de 2007.

En el presente trabajo, se proporciona una metodología para estimar el nivel medio del mar en la bahía de Paracas.

\section{Definiciones}

Una señal mareográfica puede ser el resultado de la superposición de varias componentes con diferentes frecuencias y amplitudes. Se puede representar dicha señal como una serie de Fourier de la forma

$$
y(t)=\sum_{n=0}^{N} A_{n} \cos \left(\omega_{n} t\right)+B_{n} \operatorname{sen}\left(\omega_{n} t\right) .
$$

\section{Marea}

La marea es una onda generada por el ascenso y descenso periódicos de todas las aguas oceánicas, incluyendo las del mar abierto, los golfos y las bahías, resultado de la atracción gravitatoria de la Luna y del Sol sobre el agua y de la propia Tierra. Tiene un periodo largo de más de 12 horas y una amplitud de hasta 1 metro. Es un fenómeno netamente gravitatorio [2].

\section{Olas}

Las olas son ondas superficiales generadas por el viento. Las ráfagas de viento sacuden la superficie de las aguas del mar, las rizan y dan lugar a ondulaciones que van creciendo en amplitud. Cuando el viento sopla muy fuerte, las crestas de las olas se cierran sobre sí mismas y caen formando volutas. Los vientos suaves producen aguas calmadas con ondas que pueden recorrer miles de kilómetros, y los vientos fuertes producen aguas tempestuosas. Tiene un periodo corto, alrededor de pocos segundos, y su amplitud puede llegar a varios metros como en el oleaje anómalo.

\section{Refración de olas}

Las ondas al viajar se orientan en forma paralela según sus longitudes de onda característicos, y forman los denominados trenes de ondas. Estos trenes viajan por aguas profundas hasta que se encuentran con obstáculos en su camino. Por ejemplo, al llegar a zonas de poca profundidad y chocar con bancos de arena, se produce el fenómeno de refracción de las ondas, estas desvían su trayectoria original y se orientan paralelas al obstáculo encontrado. Como los bancos de arena suelen ser alargados y paralelos a la costa, debido a las corrientes costeras, las olas se desvían e inciden de manera paralela a la playa. Otro caso de refracción es cuando existe una porción de tierra que se mete mar adentro e intercepta directamente al tren de ondas, en este caso las olas pivotean en este obstáculo y toman una nueva dirección.

Cuando las olas avanzan sobre aguas cada vez menos profundas en su camino hacia la playa, su velocidad decrece, se refractan y orientan en forma cada vez mas paralela a la orilla. Los largos bancos de arena o arrecifes de roca o coral son los obstáculos que permiten esta refracción.

\section{Maremoto}

Es un tren de ondas gravitacionales generadas por una perturbación en el mar, como un terremoto tectónico, un volcán submarino, un desplazamiento submarino o un meteorito. Tiene un periodo con valores en el rango de los varios minutos hasta una hora y la amplitud puede alcanzar hasta los 10 metros o más. El último maremoto en esta zona ocurrió el 15 de agosto de 2007. Este maremoto fue de origen local y de intensidad ligera [3.

\section{Marea de sicigia}

Se produce durante la luna nueva y llena, la amplitud de la marea aumenta en esta época pues la fuerza generadora de la marea producida por la Luna tiene dirección y sentido similares a la producida por el Sol. Para poder determinar el nivel medio del mar con una precisión casi absoluta es necesario realizar una toma de datos durante un periodo largo, durante 18.6 años, lo que corresponde a un periodo nodal lunar [2] sin embargo, con ayuda del procesamiento digital de señales se puede aproximar el cálculo empleando un menor tiempo de toma de datos.

\section{Metodología}

Para la instalación del sistema, el trabajo de campo y el trabajo de gabinete, se siguió el siguiente procedimiento:

1. Se ubicó un punto adecuado para la instalación del sensor de nivel del mar y de los equipos. Se instaló una regla mareométrica para tomar un nivel de referencia arbitrario. La instalación de los equipos se realizó el día sábado 24 de enero de 2009 en la mañana, en el muelle del Hotel Paracas, ver Fig. 1, con las coordenadas, latitud $=13.8345^{\circ} \mathrm{S}$ y longitud $=76.2551^{\circ} \mathrm{W}$. 


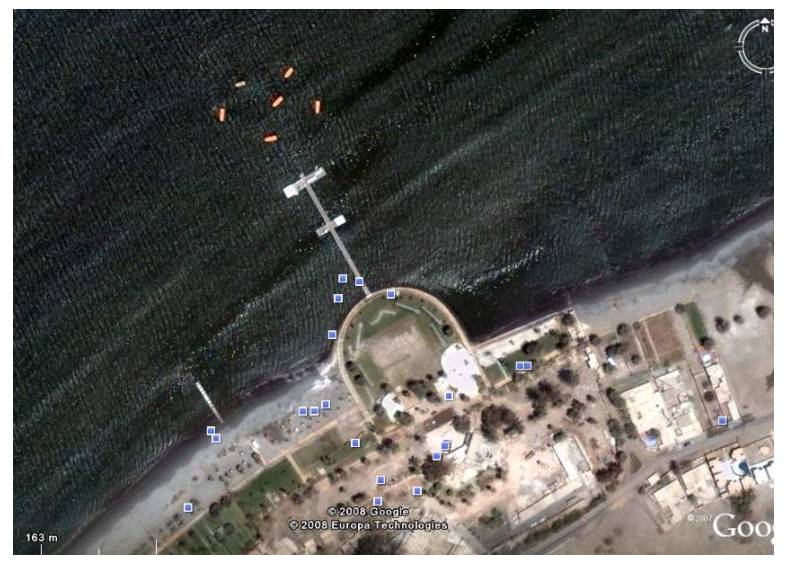

Figura 1: Imagen satelital del Hotel Paracas. Fuente: Google Earth.

2. Se ubicó un punto de fluido eléctrico y se calculó la distancia al punto de referencia donde se colocó el sensor. El punto de energía eléctrica se obtuvo de la caseta del muelle.

3. Se instaló el sensor de nivel con su respectiva interface y laptop para la adquisición de datos en forma continua y durante una semana con una frecuencia de muestreo de $1 \mathrm{~Hz}$, ver Fig. 2.

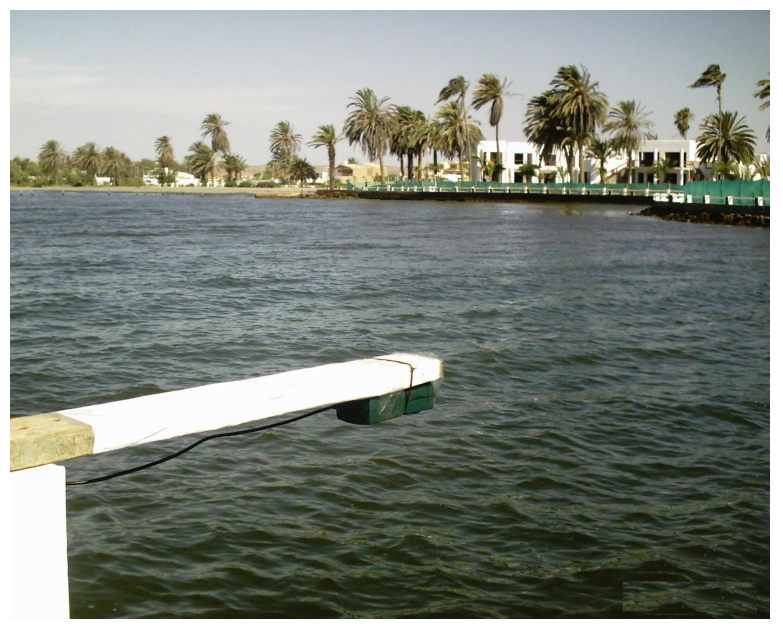

Figura 2: Sensor de nivel ultrasónico Vernier.

4. Con los datos recolectados, se realizó el procesamiento digital de la señal mareográfica para filtrar las señales indeseadas, ruido impulsivo y determinar el nivel medio del mar. Además, se obtuvo:

a) El espectro de la señal por transformada de Fourier.

b) El filtrado del ruido indeseado y las componentes de periodo muy largo mayor de un mes. c) Se encontró el valor medio de la señal y se referenció el cero arbitrario con respecto a este valor medio del nivel del mar.

5. Se realizó la nivelación utilizando un nivel de topógrafo. Se seleccionó la ubicación de los hitos, con el respectivo monumentado de dichos hitos. Se referenció el nivel de los hitos con respecto al nivel del sensor y luego con respecto al nivel medio del mar.

6. Se realizó el cálculo de la diferencia entre el nivel medio del mar y el nivel medio de bajamares de sicigias ordinarias, utilizando los resultados de la Tabla de Mareas 2009 del mareógrafo de Pisco.

7. Después del paso anterior referenciamos el nivel del hito con respecto al nivel medio de bajamares de sicigias ordinarias. Obtuvimos la posición, latitud y longitud, de los hitos utilizando un GPS.

8. Se calculó la altura del muro perimétrico, en base a los datos histórica de oleajes irregulares en la zona de Pisco y Paracas, y en base a los datos de máxima marea de la Tabla de Mareas 2009 [4].

El trabajo de campo y de adquisición de datos se concluyó el día sábado 31 de enero de 2009 a las 5:00 pm, luego se procedió a realizar copias de seguridad de los datos obtenidos y se desinstaló los equipos utilizados.

\section{Resultados}

El trabajo de gabinete empezó a partir del día lunes 02 de febrero de 2009. Se realizó el procesamiento digital de la señal mareográfica utilizando el software Logger Pro [5] y el Toolbox de procesamiento de señales de Matlab 7.0 6].

\section{Determinación del nivel medio del mar}

Lo primero que se realizó fue aplicar un filtro mediano para eliminar los picos impulsivos debido a ruido de alta frecuencia producido por los famosos vientos Paracas. Como puede verse en la Fig. 3. Según la información de los observadores de campo, estos picos se producían cuando se presentaban estos fuertes vientos.

Una vez obtenido una señal limpia de ruido indeseable, se procedió a calcular el valor medio de la señal para un periodo entero de días. Físicamente, este valor representa la longitud desde el nivel "cero arbitrario" del sensor hasta el nivel medio del mar, $L_{1}=1.75 \mathrm{~m}$. 

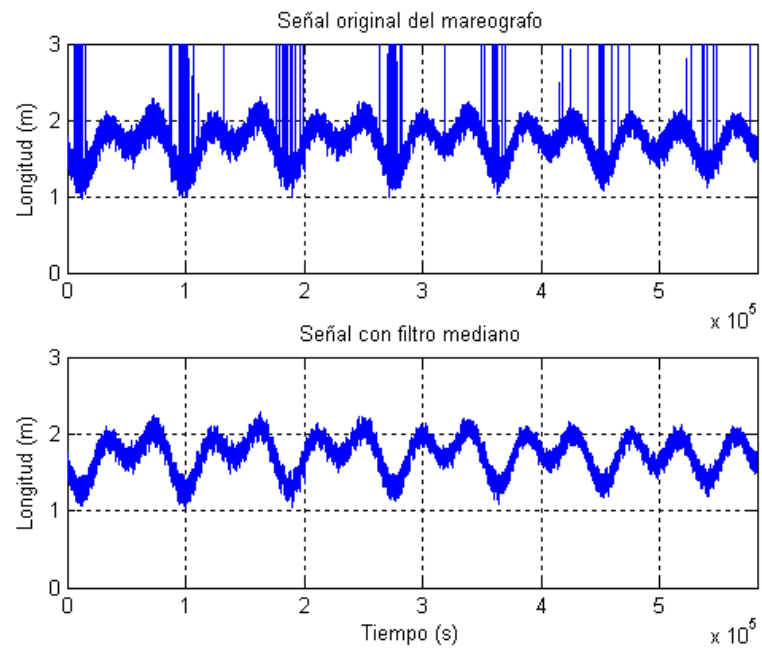

Figura 3: Eliminación del ruido impulsivo mediante filtros digitales.
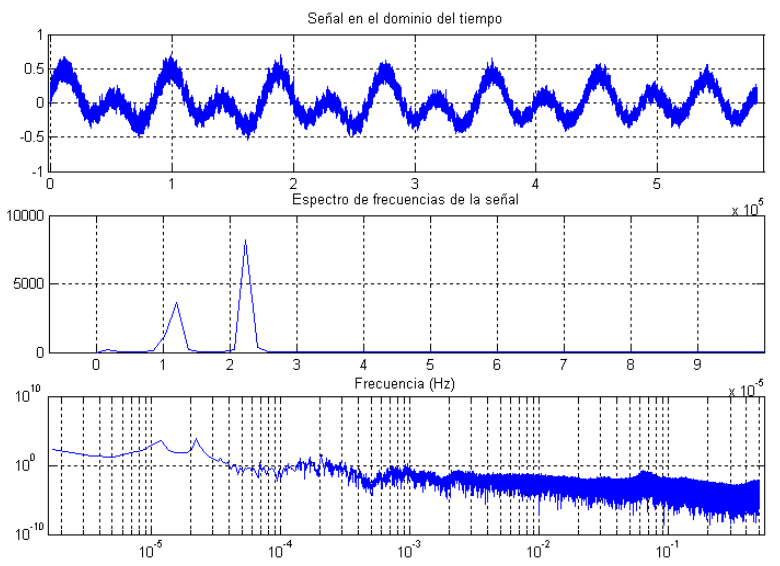

Figura 4: Señal mareográfica con su espectro de frecuencias.

Espectro de frecuencias de la señal mareográfica

La señal mareográfica real es el negativo de la salida de la señal del sensor. Una vez rectificada y eliminando la componente contínua de la señal, es decir solo considerando las componentes alternas de la señal, se procede a obtener el espectro de frecuencias lineal y logarítmica, como se puede observar en la Fig. 4. Se puede apreciar dos picos principales. Estos picos principales corresponden a un valor de frecuencia y periodo determinados, tal como se muestra en la siguiente tabla. Esto implica que hay dos componentes principales de periodo largo en la señal que se repiten alrededor de cada 12 y 24 horas prácticamente. Lo que significa que tenemos una marea semidiurna, que es un tipo de marea en la que se producen dos pleamares y dos bajamares por día.

\begin{tabular}{ccc}
\hline Pico & Frecuencia $\left(10^{-5} \mathrm{~Hz}\right)$ & Periodo $(\mathrm{h})$ \\
\hline 1 & 2.23 & 12.4 \\
2 & 1.20 & 23.2 \\
\hline
\end{tabular}

Tabla 1: Parámetros de los picos principales del espectro de frecuencias.

Determinación del nivel medio de bajamares de sicigias ordinarias

Se utilizó los valores calculados en la Tabla de Mareas 2009 (DHN), para formar una serie de tiempo o mareograma representada en la Fig. 5, con ayuda del software Matlab se calculó el valor medio de la señal. Este valor representa la distancia entre el nivel medio del mar y el nivel medio de bajamares de sicigias ordinarias, $L_{2}=0.34 \mathrm{~m}$.

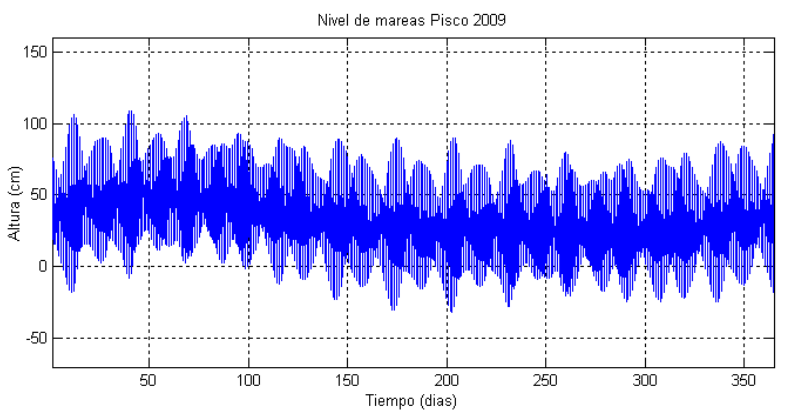

Figura 5: Mareograma de la tabla de mareas 2009 (DHN).

Nivelación de los hitos con respecto al nivel cero arbitrario del sensor

La monumentación y nivelación del hito se realizó el día viernes 30 de enero de 2009. La ubicación de dicho hito se realizó a sugerencia de los ingenieros responsables de la obra de construcción del Hotel Paracas. La nivelación respectiva se realizó con respecto al nivel cero arbitrario del sensor con un equipo de topografía marca Wild Heerbrugg FNR 542. La determinación de las coordenadas horizontales se realizó con un GPS Gar$\min$.

El nivel vertical real se realiza añadiendo las constantes $L_{1}$ y $L_{2}$ para obtener estos niveles con respecto al nivel medio de bajamares de sicigias ordinarias. Estos resultados se muestran en el parágrafo siguiente.

\section{Datos de los hitos de Paracas}

Este hito representa un nivel de referencia para la coordenada vertical 


\begin{tabular}{ll}
\hline $\begin{array}{l}\text { Hito BYP1-BM1 } \\
\text { (Bar - Lounch) }\end{array}$ \\
\hline Coordenadas \\
\hline$Z \quad=2.16 \mathrm{~m} *$ \\
Latitud $\quad=13.8352^{\circ} \mathrm{S}$ \\
Longitud $\quad=76.2548^{\circ} \mathrm{W}$ \\
$\begin{array}{ll}\text { * sobre el nivel medio de bajamares de } \\
\text { sicigias ordinarias }\end{array}$
\end{tabular}

Nota: El error inherente en la determinación de la componente vertical $Z$ es de $\pm 2 \mathrm{~cm}$. Este error depende principalmente del tiempo de adquisición de datos. Si consideramos que un año de toma de datos corresponde a un error muy pequeño de $1 \mathrm{~mm}$, para un mes el error será de $1 \mathrm{~cm}$ y para una semana será de $2 \mathrm{~cm}$.

\section{Análisis de las olas a partir de la señal mareo- gráfica}

Para estudiar las componentes de olas a partir de la señal mareográfica es necesario filtrar la componente de marea para un periodo de corte mayor que $60 \mathrm{~s}$, $f=0.0167 \mathrm{~Hz}$ y menor que $8 \mathrm{~s}, f=0.125 \mathrm{~Hz}$. De tal forma que sólo queda la componente de olas, ver Fig.6.
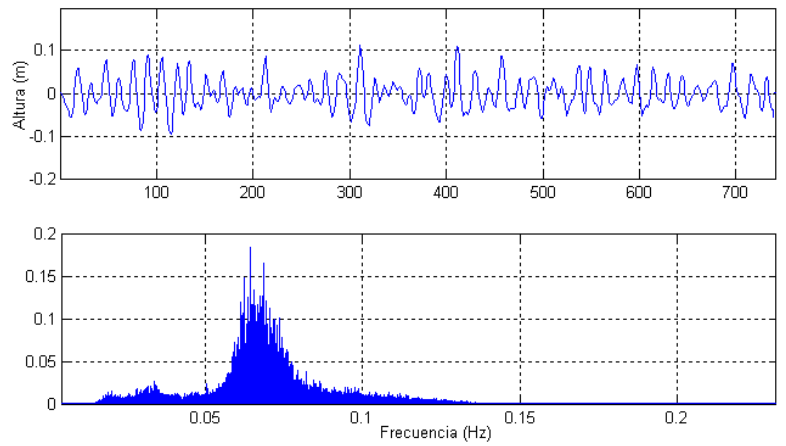

Figura 6: Registro de las olas (gráfico superior) y espectro de frecuencias (gráfico inferior).

\begin{tabular}{ll}
\hline Oleaje irregular & Amplitud \\
\hline Normal & hasta 18 \\
Ligero & $18-33$ \\
Moderado & $33-48$ \\
Fuerte & $48-63$ \\
\hline
\end{tabular}

Tabla 2: Clasificación del oleaje irregular para la zona de Pisco (DHN).

El espectro de frecuencias predominante está en el rango de $12 \mathrm{~s}$ a $18 \mathrm{~s}$. Se puede observar un pico predominante para $f=0.0642 \mathrm{~Hz}$ que corresponde a un periodo $T=15.6 \mathrm{~s}$. Entonces el tren de ondas tiene un periodo de retorno característico de alrededor de $16 \mathrm{~s}$.
La amplitud máxima, la longitud cresta-valle, de la ola registrada durante la toma de datos fue de $0.34 \mathrm{~m}$, ocurrida el día jueves 29 de enero de 2009 a las 20:47 horas. Lo que corresponde a un oleaje irregular de moderada intensidad.

\section{Cálculo de la altura del muro perimétrico}

El muro perimétrico es una referencia vertical a la cual debe edificarse cierta instalación. De la Tabla 3, podemos observar que el 06 de febrero de 1998, durante el fenómeno del Niño, se alcanzó la máxima amplitud de la ola, $68 \mathrm{~cm}$, en un periodo de oleaje irregular de intensidad muy fuerte.

Según la información de la Tabla de Mareas 2009, la más alta marea calculada es de $1.09 \mathrm{~m}$ sobre el nivel medio de bajamares de sicigias ordinarias.

En base a estos datos de máxima marea y de máximo oleaje podemos estimar la altura del muro perimétrico, como la suma de estas cantidades más un factor del $10 \%$ del valor para tener en cuenta alguna perturbación esporádica adicional o alguna anomalía,

$$
H=(0.68+1.09)+10 \%(0.68+1.09)=1.95 \mathrm{~m} .
$$

\begin{tabular}{lccl}
\hline Fecha & Hora & Amp $(\mathrm{cm})$ & Intensidad \\
\hline 98 may 29 & $13: 00$ & 61 & Fuerte \\
98 Feb 06 & $17: 55$ & 68 & Muy Fuerte \\
99 Mar 06 & $11: 10$ & 52 & Fuerte \\
00 Feb 19 & $21: 00$ & 49 & Fuerte \\
01 Jun 23 & $22: 42$ & 43 & Moderado \\
06 May 30 & $12: 00$ & 46 & Moderado \\
08 Jul 17 & $17: 38$ & 40 & Moderado \\
\hline
\end{tabular}

Tabla 3: Oleaje irregular histórico medido por el mareógrafo de Pisco (DHN).

Luego, la estimación de la altura del muro perimétrico es $H=1.95 \mathrm{~m}$ sobre el nivel medio de bajamares de sicigias ordinarias.

\section{Conclusiones}

Las conclusiones de mayor relevancia, obtenidas del desarrollo del trabajo son las siguientes:

El nivel medio del mar no es un parámetro constante, sino que es variable en el tiempo y depende de varios factores como: variaciones del campo gravitatorio, ocurrencia de fenómeno de El Niño, cambio climático a nivel global, movimientos sísmicos locales, etc. Sin embargo, estos son eventos de periodo largo, en el orden de tiempo de varias décadas. Se ha determinado una metodología para calcular el nivel medio del mar en base a toma de datos mareográficos y realizando el procesamiento digital de la señal. 
La importancia de conocer este parámetro, nivel medio del mar, surge de la necesidad de la construcción de obras de infraestructura portuaria y edificaciones cerca al mar, que requieren un nivel de referencia vertical bien determinado.

Si consideramos que para un año de toma de datos el nivel medio del mar obtenido es cuasi exacto, para un mes de toma de datos se tendrá un error de $1 \mathrm{~cm} \mathrm{y}$ para una semana se tendrá un error de $2 \mathrm{~cm}$. Esto es inherente a la naturaleza estadística del problema.

El resultado obtenido es válido para un periodo nodal lunar, equivalente a un poco más de 18 años.

Debido a un terremoto de gran magnitud, $\mathrm{Mw}>7.0$, todo el marco de referencia podría variar debido al desplazamiento co-sísmico de las placas tectónicas. Por lo tanto, el resultado obtenido será válido hasta el próximo gran terremoto en la región de Pisco-Paracas.
Según la información de la Tabla de Mareas 2009 la más alta marea calculada es de $1.09 \mathrm{~m}$ sobre el nivel medio de bajamares de sicigias ordinarias. El máximo oleaje registrado por la estación mareográfica de Pisco tuvo una amplitud de $68 \mathrm{~cm}$ en el año de 1998.

No se ha considerado la vibración de la estructura del muelle debido a que son oscilaciones de pequeña amplitud y de periodo corto, los mismos que son filtrados por el sistema de adquisición de datos.

\section{Agradecimientos}

Se agradece a Pedro Bergelund de la empresa ByP Operaciones por financiar el presente proyecto y al personal del Hotel Paracas por brindar el apoyo logístico y de seguridad al personal y a los equipos de medición.

\section{Referencias}

[1] L. Ocola; Aspectos físicos del maremoto de Pisco del 15 de agosto de 2007 y las inundaciones máximas, del libro El terremoto de Pisco del 15 de agosto de 2007 (7.9 Mw), Instituto Geofísico del Perú, Lima (2007).

[2] David Pugh; Tides, Surges and Mean Sea Level, John Wiley \& Sons, Londres (1996).

[3] E. Ortega y C. Jiménez; Revista de Tsunamis, Di- rección de Hidrografía y Navegación (DHN), Lima (2010).

[4] DHN; Tabla de mareas, Dirección de Hidrografía y Navegación de la Marina de Guerra del Perú, Hidronav, Lima (2009).

[5] Recurso electrónico; Software Logger Pro, version 3.7, página web: http://vernier.com

[6] Signal Processing Toolbox User's Guide, version 4.2 The MathWorks Inc. (1999). 